\title{
Guest Editorial: Special Issue on Parameterized and Exact Computation
}

\author{
Gregory Z. Gutin • Stefan Szeider
}

Received: 14 November 2014 / Accepted: 19 November 2014 / Published online: 4 December 2014 (C) Springer Science+Business Media New York 2014

This issue is dedicated to a selection of papers from the the 8th International Symposium on Parameterized and Exact Computation (IPEC 2013), held during September 4-6, 2013, in Sophia Antipolis, France, as part of the annual ALGO meeting. Nine papers were submitted to this special issue and went through the standard refereeing process of Algorithmica.

The paper "On Subexponential and FPT-time Inapproximability" by Edouard Bonnet, Bruno Escoffier, Eun Jung Kim, and Vangelis Th. Paschos provides new insights into the question of whether the INDEPENDENT SET problem would be better approximable once endowed with subexponential-time or FPT-time. The paper "Multiparameter analysis for local graph partitioning problems: using greediness for parameterization" by Édouard Bonnet, Bruno Escoffier, Vangelis Th. Paschos, and Émeric Tourniaire develops the new algorithmic technique of "greediness for parameterization" which applies to local graph partitioning problems. The paper "Incompressibility of $\mathrm{H}$-Free Edge Modification Problems" by Leizhen Cai and Yufei Cai studies the kernel complexity of problems that ask whether one can add or delete $k$ edges from a given graph so that the graph does not contain a fixed graph $H$ as an induced subgraph.

The paper "On the Space and Circuit Complexity of Parameterized Problems: Classes and Completeness" by Michael Elberfeld, Christoph Johannes Stockhusen, and Till Tantau shows that various natural problems lie in and may even be complete for different parameterized space classes which leads to new insights into the complexity of the considered problems. The paper "Speeding Up Dynamic Programming with Representative Sets-An Experimental Evaluation of Algorithms for Steiner Tree on Tree Decompositions" by Stefan Fafianie, Hans L. Bodlaender, and Jesper Nederlof provides an experimental evaluation of the technique of representative sets for the

G. Z. Gutin $(\varangle) \cdot$ S. Szeider

University of London, Egham, UK

e-mail: gutin@cs.rhul.ac.uk 
STEINER TREE problem on graphs of small treewidth. The paper "A Completeness Theory for Polynomial (Turing) Kernelization" by Danny Hermelin, Stefan Kratsch, Karolina Sołtys, Magnus Wahlström, and Xi Wu defines two kernelization hardness hierarchies and establishes the completeness of various problems for classes in the new hierarchies.

The paper "On Sparsification for Computing Treewidth" by Bart M. P. Jansen shows that the problem of deciding whether a given graph has treewidth at most $k$ does not admit a sparsification unless the polynomial hierarchy collapses. The paper "Obtaining Matrices with the Consecutive Ones Property by Row Deletions" by N. S. Narayanaswamy and Subashini R. Subashini studies the problem of deciding whether one can delete $d$ rows from a binary matrix so that afterwards the matrix has a permutation of columns that arranges the ones consecutively in all the rows, and introduces a closely related optimization problem. The paper "Pure Nash Equilibria in Graphical Games and Treewidth" by Antonis Thomas and Jan van Leeuwen explores the role of treewidth the problem of deciding the existence of a Pure Nash Equilibrium in a graphical game.

We would like to thank the authors for contributing to this special issue and the referees for their attentive and helpful work in the reviewing process.

Guest Editors 